\title{
Editorial for special issue of Telecommunication Systems on "Mobility management and wireless access"
}

\author{
Sherali Zeadally • Albert Zomaya • Han-Chieh Chao
}

Published online: 7 July 2009

(C) Springer Science+Business Media, LLC 2009

In the last decade, we have been witnessing an explosive growth in the development, deployment, and adoption of a wide range of wireless communication technologies and applications. Ubiquitous access of information anywhere, anytime, from any device by end-users continues to drive the need to develop innovative design architectures, protocols, and networks with capabilities that can help achieve this goal. The advent of mobile networks and applications has opened up many research challenges that need to be addressed to enable seamless end-to-end communication transparently over a highly heterogeneous network infrastructures. In particular, mobility management and wireless access are two specific areas where a lot of attention has been given to in order to achieve the seamless end-to-end connectivity goal. This special issue is dedicated to original results and achievements by active researchers, designers, and developers working on various issues and challenges related to mobility management and wireless access. After a rigorous peer review process we accepted ten papers (with authors from twelve countries). These papers cover a wide range of topics including mobility management and protocols, modeling, performance analysis, Quality of Service (QoS), and scheduling.

In "Access selection and mobility management in a beyond 3G RAN: the WINNER approach", Tragos et al.

\section{S. Zeadally $(\bowtie)$}

University of the District of Columbia, Washington DC, USA

e-mail: szeadally@udc.edu

\author{
A. Zomaya \\ University of Sydney, Sydney, Australia \\ H.-C. Chao \\ National Ilan University, Ilan, Taiwan
}

present a flexible mobility management architecture that supports multiple operating modes for wireless access in the local, metropolitan, and wide area environments. To address the limitations of centralized and distributed admission control schemes, the authors propose hybrid handoff and admission control approaches by introducing a radio resource management server in their proposed architecture. Low network cost and reduced complexity are some of the major benefits of their proposed mobility management design architecture.

In "Towards the Formation of Comprehensive SLAs between Heterogeneous Wireless DiffServ Domains", Iftikhar et al. present novel analytical models based on the G/M/1 queueing system using different classes of self-similar traffic to model wireless IP traffic behavior. The results of this work are useful in bandwidth and buffer allocations to support guaranteed end-to-end QoS. Moreover, this work also provides some concrete results that help us to better understand the end-to-end QoS behavior of different types of traffic classes when delivered over heterogeneous wireless IP domains.

High mobility in ad hoc networks often causes significant disruptions in service delivery because of frequent loss of connections and their re-establishments. Previous researchers have addressed this issue by focusing mainly on reactive protocols based on signal strength measurements. As a result, significant delays are introduced between link failure and the re-establishment of the link when a mobile node moves. In "Signal Strength Based Link Sensing for Mobile Ad-Hoc Networks", Ali et al. propose a proactive protocol based on the Optimized Link State Routing (OLSR) protocol that improves the robustness of link management protocol by determining if the link quality is improving or deteriorating. By avoiding loops in the network, the proposed approach improves network performance. 
The low output power and high data rate provided by Ultra Wide Band (UWB) make this technology popular for wireless personal area networks. Both single and multi-band UWB systems have been proposed in the literature and several past efforts have studied the performance of UWB systems. In "Comparisons of Various Pulse Shapes for DSUWB Signals over the UWB Channel", Ozturk et al. investigate the appropriate values of pulse durations of the derivatives of the Gauss pulse for Direct Sequence Ultra Wide Band (DS-UWB) signals and present probability error results for the CM1 channel model.

Wireless multimedia applications involving continuous media such audio and video have become very popular. The IEEE 802.11e standard has been developed to specifically address QoS support for multimedia traffic. In "An Adaptive Cross-layer Mapping Algorithm for MPEG-4 Video Transmission over IEEE 802.11e WLAN", Lin et al. present an adaptive, cross-layer mapping algorithm aimed at improving MPEG-4 video transmission over IEEE 802.11e networks. Simulation results obtained demonstrate not only the proposed algorithm can better prioritize video traffic based on hierarchical video information but also improves queue length utilization at the media access control layer.

The Received Signal Strength Indicator is often used to estimate the position of static and mobile wireless objects. In "Evaluation of Impact Factors on RSS Accuracy for Localization and Tracking Applications in Sensor Networks", Stoyanova et al. investigate the accuracy and reliability of received signal strength data in determining the actual location of a mobile object. They conduct actual real-field outdoor environments to study the effect of various parameters (such as operating frequency, antenna orientation, etc.) on the received signal strength. Such results will be useful in the development of future receiver signal strength localization and tracking techniques.

In "State-Dependent Packet Scheduling for QoS Routing in a Dynamically Changing Environment", Bourenane et al. investigate the problem of optimizing the queueing delay introduced by routers of a network through a global packet scheduling approach that has the capability of adapting to new traffic conditions. To achieve this global objective, a decentralized, multi-agent system which makes use of antlike mobile agents deployed on each router is exploited to guarantee a global view of the system's state. The simulation results obtained with the modified Q-learning algorithm demonstrate the effectiveness of their proposed approach.
The last three papers deal with mobility protocols such as Mobile IPv4 and Mobile IPv6 which have been developed to support user mobility across IP-based networks. In "A Terminal-Assisted Route Optimized NEMO Management", Lim et al. propose a novel terminal-assisted IP mobility protocol that enables mobile network nodes to work with mobile access routers to provide seamless connectivity. This protocol eliminates the pinball routing effect associated with NEMO and eliminates the need of other entities such as home agents. In "ILNP: Mobility, Multi-Homing, Localised Addressing and Security Through Naming", Atkinson et al. propose a naming architecture intended to address some of the limitations of mobility protocols such as Mobile IPv4 and Mobile IPv6. An instance of the proposed architecture and a new network-layer protocol based on IPv6 are presented. The main strength of their proposed solution is that its deployment does not require modifications of currently deployed IPv6 routers and can easily evolve over existing network infrastructures. In "An Efficient Mobile IPv6 Handover Scheme", Chen et al. propose a novel handoff method called Seamless Handover for Mobile IPv6 (S-MIPv6). The main goal of their proposed method is to improve the handoff performance. This is achieved by avoiding the need to establish tunnels and by minimizing registration delays. Their simulation results demonstrate that both soft and hard mode S-MIPv6 can perform better than the Fast Handover for Mobile IPv6 (F-MIPv6) approach in terms of both handover latency and packet loss.

We would like to take this opportunity to thank the Editor-in-Chief of the Telecommunication Systems Journal, Professor Bezalel Gavish, for his invaluable support and encouragements throughout the preparation of this special issue. We thank the staff at Springer for their kind help, and in particular Jackie James from Southern Methodist University. We express our deepest gratitude to all the anonymous reviewers who devoted much of their precious time reviewing all the papers submitted to this special issue. Their timely reviews greatly helped us select the best papers included in this issue. We also thank all authors who contributed to this special issue.

Finally, we hope you will enjoy reading this selection of papers as we did and you will find this issue informative and helpful in keeping yourselves up-to-date in the fast changing fields of mobility management and wireless access. 